\title{
Blomechanics
}

\section{Upgrading of efficiency in the tracking of body markers with video techniques}

\author{
C. J. Keemink G. A. Hoek van Dijke C. J, Snijders \\ Doparment of Biological and Medical PhVsics, Erasmus Unversity Romerdam. \\ PO Box 1738, 3000 OR Rotrerdin, The Notherands
}

\begin{abstract}
Based on a VWE system, a low - cost Video system has been developed for recerding human motion. The paper describes the algrorith which is used for the recordings. The video system makes it possible to track in real time up to sik markers. on the body. sampled at a $50 \mathrm{hz}$ rate:
\end{abstract}

Keywords-Biomechanics, Gait analysis, Human motion, Video

Med. 8. Bial Eng. 8. Compur. 1991, 29, 70-74

\section{Introduction}

FOR THE evaluation of an ankle orthosis we were in need of a method of recording leg movements during walking. Several techniques are described in the literature. Electromechanical transducers, as used by IsACSON et al. (1986) and OLsSON et al. (1986), produce electric signals which are suitable for automatic data processing, but the attachment of transducers and wires to the body can interfere with human motion. The Selspot system, which is used by NASHER and FORSSBERg (1986) and THORSTENSSON (1986), makes contactless recording possible. This system, however, is sensitive to interfering light and the subject must be connected by wires to the apparatus, which can be troublesome in some cases. Recording human motion without connecting any equipment to the subjects is possible by means of a film technique, as used by HAGEMAN and Blanke (1986) and Olney et al. (1986). A disadvantage is that no electric output is generated, which hampers automatic data processing. Video techniques can combine the advantages of contactless recording and automatic data processing. However, the sample rate is limited.

As it was available to us, we used a video system for our recordings. Reflective markers were attached to the leg. Light was projected on to the markers from the direction of the camera, causing them to produce bright images. The markers can be distinguished from the rest of the image by means of a luminance threshold. The threshold value is so chosen that it is exceeded only by the images of the markers. The position of each marker is then determined in every video field. In this paper we introduce the FIR (fast image recognition) algorithm which we used for the real-time analysis of the video recordings. This algorithm enabled us to track a sufficient number of markers, using a general-purpose computer. The algorithm is suitable for tracking markers whose motions are in some way related to each other, as is the case with most human motions.

First received 26th June 1989 and in final form 26th February 1990 (C) IFMBE: 1991

\section{Methods and materials}

The method described in this paper has been developed for the analysis of the side view of human gait. The leg is represented as three rigid links. The positions of these links can be determined by recording two fixed points on each link. Six round markers are therefore attached to the subject as shown in Fig. 1. The subject walks on a moving belt, making it possible to record the side view of the motion pattern for a considerable period of time with a stationary camera. We used a CCD camera (Sony AVCD5CE) and stored the recordings on videotape.

For the analysis of the tape we used a VME computer system, based on a 68000 processor and a separate memory board with $512 \mathrm{k}$ byte RAM. In addition to this basic equipment we had two boards with video facilities, manufactured by Datacube: a Digimax-7 digitiser and display module, which digitises the video signal (256 grey levels), and a Framestore-7 triple frame storage module, which can store two video frames in two independent memories ( 512 lines, 384 pixels per line).

A video frame consists of two fields: one field encloses the odd video lines, the other encloses the even lines. Both fields contain information about the whole picture, but the spatial resolution in a vertical direction is only half of the resolution of a video frame. The two fields are refreshed alternately. We used the information in each separate field, which enabled us to achieve a $50 \mathrm{~Hz}$ sample rate (CCIR norm).

The computer is programmed to store the fields alternately in the first and second video memory. The analysis of a field in one of the memories takes place during the building up of the next field in the other memory. In both memories, building up a field and analysing a field alternate, so one field time $(20 \mathrm{~ms})$ is available for analysing each field.

The lookup table of the Digimax board is programmed to digitise the video signal to obtain a two-level picture: the body markers become white, the rest of the picture becomes black. The real-time analysis of the pictures is 
confined to calculating the $x$ and $y$ co-ordinates of the centre of each marker. To do this, a matrix of points is scanned to detect an arbitrary point within each marker. The distance between these matrix points is as great as possible without allowing a marker to fit between them (Fig. 2).

Once an arbitrary point of a marker has been found, the centre of the marker is calculated. The use of circular markers makes it possible to find the centre quickly (Fig. 3). On the horizontal line on which the first point $P$ of the marker was found, pixels to the right and left of that point are scanned to detect the boundary points $h_{1}$ and $h_{2}$. Midway between these points is the vertical diameter of the marker. The centre of the marker can be determined by calculating the middle point of this vertical diameter.

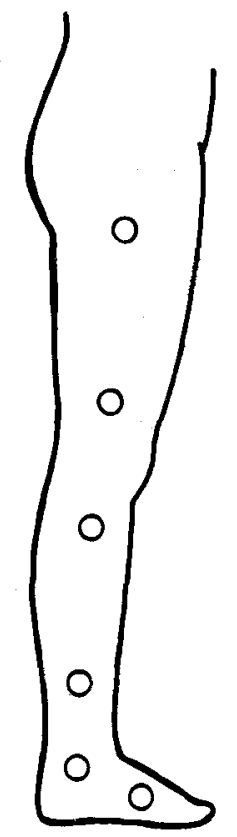

Fig. 1 Marker positions on the leg

However, in practice it is found that, because the image is not precisely round, the calculated centre can deviate from the real centre in a horizontal direction when the first point $P$ is located close to the upper or lower rim of the marker. The pixels on the horizontal line on which the calculated centre is situated are therefore scanned to detect the middle point of this line. This middle point is then taken as the centre of the marker.

Our computer system is not fast enough to scan a matrix as big as a whole picture. That is why an algorithm was introduced which restricts scanning to those parts of the picture in which a marker can be expected to be found. The algorithm is based on prior knowledge regarding the motion pattern. In our case this was the gait pattern of a subject walking on a moving belt.
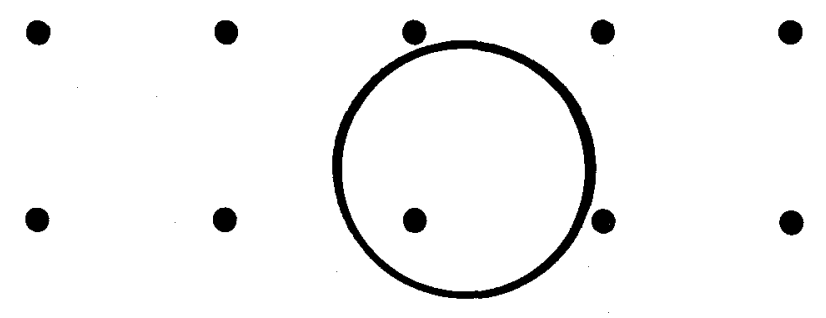

Fig. 2 The distance between the matrix points must be as great as possible, but such that a marker cannot fit between them
Fig. 4 illustrates the scanned matrix points in an arbitrary field. During walking, the marker on the hip moves only in a small area. This marker can be found very quickly by scanning a small rectangular matrix at the top of the picture. The next marker, just above the knee, always moves in a restricted area beneath the hip. To find this second marker it is sufficient to scan a triangular matrix below the first marker. Because of known minimum distance between these markers, the top of the triangle can be skipped. We skipped a fixed number of matrix rows,

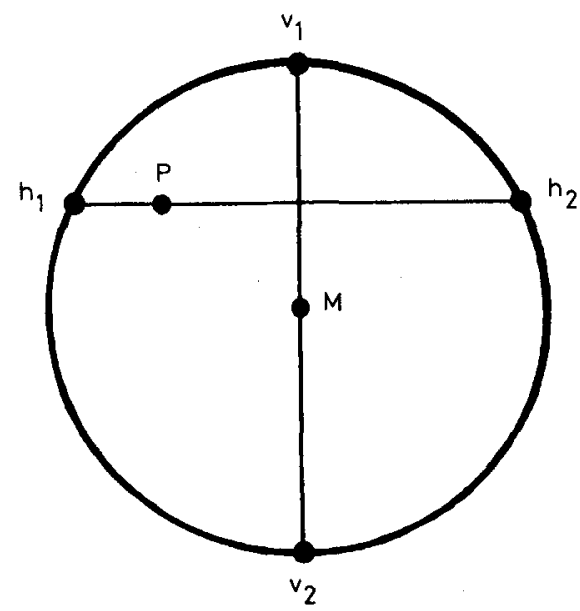

Fig. 3 Calculation of the centre of a marker. From an arbitrarily found point $P$, the points $h_{1}$ and $h_{2}$ on the edge of a marker image are determined by scanning pixels in a horizontal direction. Midway between these points, pixels are scanned in a vertical direction to determine the points $v_{1}$ and $v_{2}$. The centre of the marker is midway between $v_{1}$ and $v_{2}$. To prevent errors as a result of inexact images, at the level of point $M$ pixels are scanned in a horizontal direction once more to determine the edges of the image. The position midway between these edges is taken as the centre of the marker

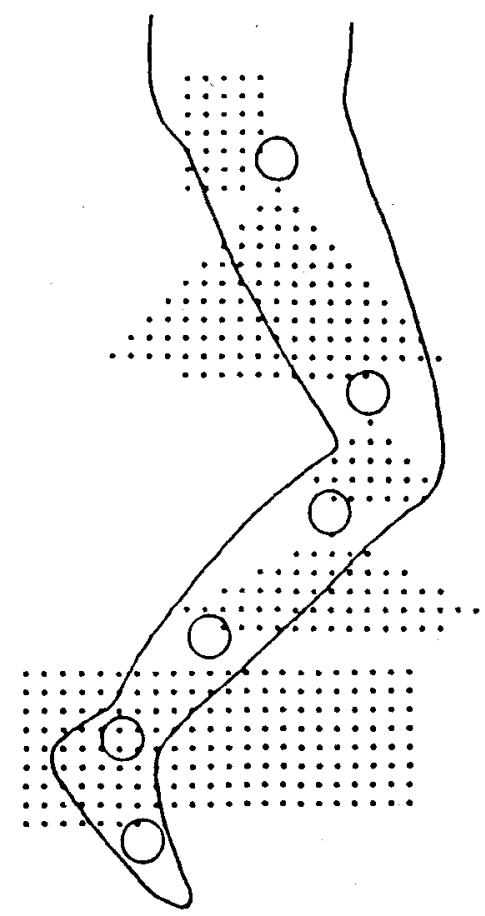

Fig. 4 After determining the top marker, each marker can be found in a restricted area below the previous marker. This figure shows the strategy for determining the positions of the markers, moving downwards from the marker on the hip. Each dot represents a scanned position. The starting position for searching for a marker depends on the position of the previous marker 
and a suitable number of matrix rows was determined for each trial. The markers below all move in a restricted area in relation to the previous marker. All the time, only a matrix of limited dimensions need be scanned to find the next marker. The starting position of every matrix is dependent on the position of the previous marker. For the side view of gait, it was found that, successively, a rectangle, a triangle with a small apical angle, two triangles with large apical angles and again a rectangle were appropriate shapes.

\section{Considerations in determining the optimum search strategy}

\subsection{Shape of the markers}

The use of circular markers makes it possible to calculate the centre of the markers by means of a simple algorithm, regardless of the orientation of the markers.

\subsection{Distance between matrix points}

To minimise the number of matrix points to be scanned, the distance between the points must be as great as possible. However, it must not be greater than $1 / \sqrt{2}$ times the diameter of the markers. If it were, it would be possible for a marker to fit between the matrix points, in which case it would be unfindable (Fig. 5). A distance between matrix points of half the diameter of the markers was found to be satisfactory.

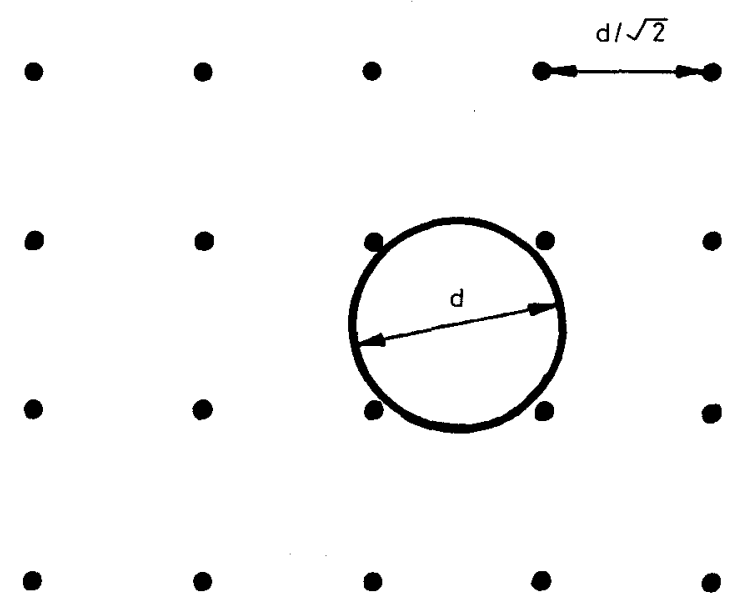

Fig. 5 The distance between matrix points must be smaller than $1 / \sqrt{2}$ times the marker diameter. A greater distance would allow a marker to fit between the matrix points, in which case it would be unfindable

\subsection{Shape of the matrices}

The shape of the area in which a marker is searched for must enclose the area in which that marker can move, leaving a margin which is as small as possible. However, the algorithm which calculates the next position must not be too complex, otherwise the calculation will take too long. For example, as the markers on the lower limb rotate around the knee, the obvious course would seem to be to search for them along an arc (Fig. $6 a$ ). Calculating position on an arc takes so much time, however, that it is faster to scan a few more points in straight lines (Fig. 6b).

\subsection{Size of the matrices}

By limiting the size of the matrices a situation is achieved in which the search for a marker stops when the marker is temporarily not visible in a field.
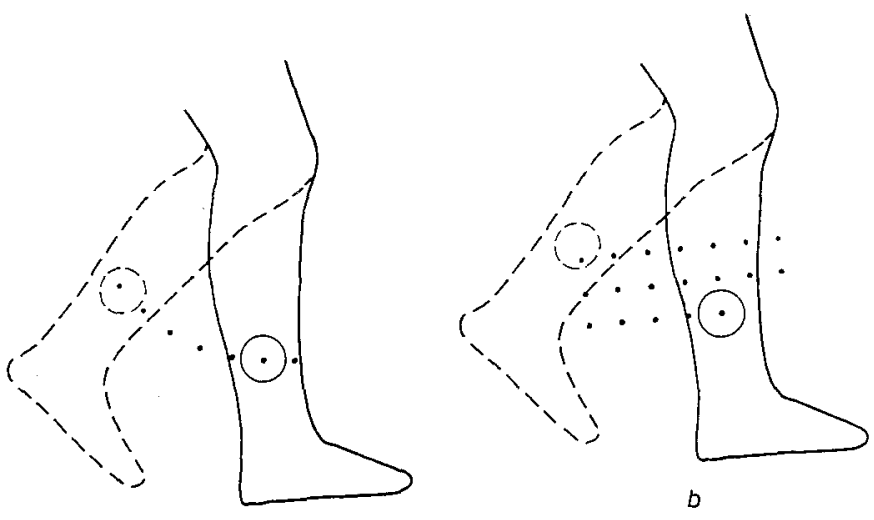

a

Fig. 6 Where markers move in a circular path around a point, it seems obvious to search for them along an arc (a). Calculating positions on an arc takes so much time, however, that scanning a greater number of matrix points in a rectangle can be faster (b)

\subsection{Number of markers to be found within the same matrix}

When two or more markers have to be found within the same matrix, it is possible that one marker will be found twice via different matrix points. As calculating the centre of a marker takes up valuable time, it is not advisable to verify that two markers found are really different markers by means of the calculated centres. Instead, we use the large distance between the markers in relation to their diameters. After a marker is detected, an area is defined all round it. Inside this area new white pixels are ignored. As this check takes extra time, if possible it is advisable to select matrices in which only one marker can be found.

\subsection{Starting point of matrices}

In our case, the motion of one of the markers was small. This marker could be found from a fixed starting point. The starting point for the search for the other markers was dependent on the position of the previous marker. The starting point for the new matrix must be far enough away from the previous marker to avoid a white pixel of that marker being found.

\subsection{Strategy within a matrix}

A proper search strategy within a matrix can save time. The mean time required to find the marker will be smaller if the matrix is scanned from the middle outwards, as this avoids scanning of the margins of the matrices.

\subsection{Distance between the markers}

The number of matrix points to be scanned can be very great if two markers are placed far apart. Sometimes an
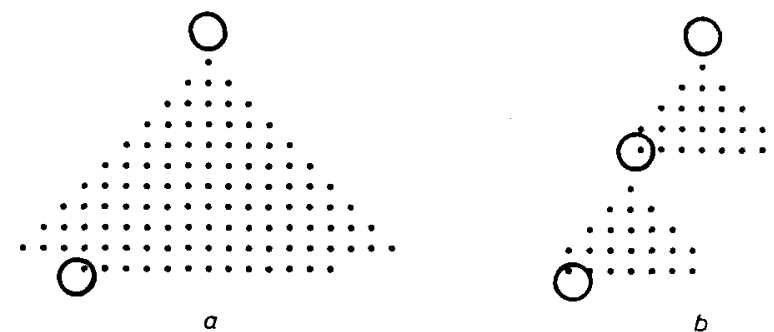

Fig. 7 The number of matrix points to be scanned can be very great where two markers are far apart (a). The number of points can be reduced by adding an extra marker between these markers (b) 
additional marker between these two markers can reduce the number of matrix points, because this divides the large matrix into two smaller matrices (Fig. 7). To save time, the calculation of the centre of this additional marker can be omitted.

\subsection{Searching outside the picture}

If a marker can come close to the edge of the picture, there is a danger that part of the matrix will be off the picture. This means that matrix points are scanned outside the video memory or in memory which does not exist. To avoid this, the positions of matrix points have to be checked to ensure that they are inside the picture. As this takes extra time, it is advisable to select matrices which can only move within the video picture.

\section{Results}

An estimation of the time required showed that it takes about $0.1 \mathrm{~ms}$ to scan a single matrix point. Once an arbitrary point of a marker has been found, it takes about $0.5 \mathrm{~ms}$ to calculate the co-ordinates of the centre. Using a $20 \mathrm{~ms}$ field time, this means that a maximum of 200 matrix points can be scanned. For each calculation of a marker's centre, five matrix points have to be subtracted from this total. Systematically scanning the whole picture, we were able to track only three markers which were allowed to move in half of the picture. The diameter of the markers was $1 / 25$ th of the height of the picture. Using the FIR algorithm, we are able to track six markers which can move on the whole picture. This algorithm multiplies both the number of markers and the area in which they can move by a factor of two.

\section{Discussion}

The FIR algorithm is suitable for image-processing systems which temporarily store video pictures in a memory. Only one field time is available for the analysis of each field, after which the video memory is refreshed. As the algorithm uses prior knowledge regarding the motion pattern which is to be analysed, its applicability depends on the degree to which such knowledge exists.

We used the algorithm for the analysis of a gait pattern. In view of mechanical theory there should not be a distinction between overground walking and walking on a moving belt (VAN INGEN SCHENAU, 1980). However, the change in perception may disturb the subject's natural gait. Research by BoLDINGH et al. (1984) showed no differences in timing and stride dimensions of gait, provided that the belt speed was chosen equal to the subject's free speed on a free walkway.

It is fairly simple to make the system insensitive to interfering light. Interfering images outside the matrices do not have any influence on the process. A check can be built in to determine whether an image is a marker or not. In our study, troublesome reflections occurred from some parts of the orthosis. These reflections were smaller than the markers, so they could be distinguished from markers by means of their diameters. A reffection was ignored if its diameter was too small. In view of the extra time which this takes, it is advisable to adjust the exposure carefully to avoid the necessity of such checks.

Errors occur in calculating the centres of the markers if the images blur at high speeds. The reason is that the circular shape of the images, on which the calculation is based, is lost. Where this happens, calculating the centre of gravity would be a better alternative, but it takes too much time. One possibility for improving the accuracy of the calculation is stroboscopic exposure, which preserves the circular images of the markers. A more expensive solution is to use a camera with an electronic shutter. Our camera was equipped with an interline sensor, which integrates light during a whole frame time $(40 \mathrm{~ms})$. The maximum rate for stroboscopic exposure is therefore only $25 \mathrm{~Hz}$. Other types of sensors such as the frame transfer sensor, which integrate light during only $20 \mathrm{~ms}$, make stroboscopic exposure at a $50 \mathrm{~Hz}$ rate possible.

The algorithm which calculates the co-ordinates of the centre of a marker is based on circular images, which are symmetric on their vertical and horizontal midline. However, such an image will be an ellipse if the marker rotates out of the sagittal plane, e.g. by abduction at the hip. When this rotation takes place on a vertical or horizontal axis, the image remains symmetric and no error is introduced. However, rotation on other axes result in erroneous calculations of the centre of the markers. The major error occurs when the axis of rotation and the horizontal direction meet at an angle of $45^{\circ}$, but small rotations have little effect. Rotations up to $30^{\circ}$ cause the calculated centre to deviate less than $2 \%$ of the diameter of the marker, in case of $45^{\circ}$ rotation this deviation becomes 10 per cent. For our research, concerning the side view of a gait pattern, the deviation of the calculated centre of the markers caused by rotation out of the sagittal plane is estimated at one pixel.

Errors in the synchronisation of the camera and the computer cause noise in the representation of the camera plane in the video memory. Calculations are estimated to deviate one pixel due to this error source. The use of cameras which can be read out pixel by pixel, using an external pixel clock, may overcome this problem.

Owing to the fact that one of the markers made only small movements, we could find this marker in every field starting from a fixed position. In cases where all the markers can move over a larger area, one field can be used to find the first marker. In the following fields all markers can be looked for, starting from the position of the first marker in the previous field. A disadvantage of this principle is that the program can get stuck when the first marker is temporarily not visible.

\section{Conclusions}

The FIR algorithm which has been described here can be used to increase the speed of video image processing This enables relatively simple equipment to be used for certain types of recordings. The applicability of the algorithm depends on the amount of prior knowledge regarding the motion pattern to be analysed.

\section{References}

Boldingh, E. J. K., Hermans, J., van Pijkeren, T. and WiJkMANS, D. W. (1984) De invloed van een tapis roulant op het looppatroon (in Dutch). Ned. Tijdschr. v. Fysioth., 94, 234-237.

HAGEMAN, P. A. and Blanke, D. J. (1986) Comparison of gait of young women and elderly women. Phys. Ther., 66, 1382-1387.

IsACSON, J., LeNNART, G. and KNUTSSON, E. (1986) Threedimensional electrogoniometric gait recording. J. Biomech., 19, $627-635$.

NASher, L. M. and ForssberG, H. (1986) Phase-dependent organization of postural adjustments associated with arm movements while walking. $J$. Neurophysiol., 55, 1382-1394.

Olney, S. J., Monga, T. N. and Costigan, P. A. (1986) Mechanical energy of walking of stroke patients. Arch. Phys. Med. Rehabil., 67, 92-98. 
Olsson, E., ÖBERG, K. and RIBBE, T. (1986) A computerized method for clinical gait analysis of floor reaction forces and joint angular motion. Scand. J. Rehab. Med., 18, 93-99.

THORSTENSSON, A. (1986) How is the normal locomotor program modified to produce backward walking?. Exp. Brain Res., 61, 664-668.

van Ingen Schenau, G. J. (1980) Some fundamental aspects of the biomechanics of overground versus treadmill locomotion. Med. \& Sci. Sports \& Exerc., 12, 257-261.

\section{Authors' biographies}

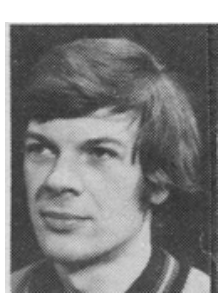

C. J. Keemink was born in Poortugaal, The Netherlands, in 1941. He studied Electronics. Since 1969 he has been working at the Erasmus University in Rotterdam. Until 1988 his research area was the analysis of eye movement by means of video techniques. At present he is working in the field of biomechanics, using video techniques for recording human motion.

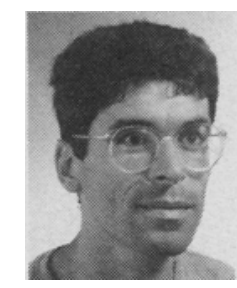

G. A. Hoek van Dijke was born in 1959 and studied Mechanics at the University of Delft, The Netherlands. In 1988 he joined the Department of Biological \& Medical Physics of the Erasmus University Rotterdam. His main research interests are in the field of recording human motion as a method for evaluating prosthetic devices.

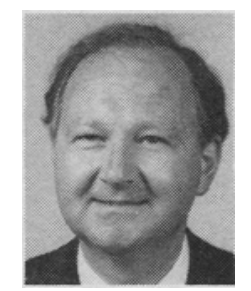

C. J. Snijders graduated in mechanics in 1966 . His thesis in 1970 regarded a study on the mechanical behaviour of the human spine. As a scientific staff member of the University of Eindhoven and Twente he was involved in research, product design and lecturing related to the medical Departments of Cardiology, Radiology, Orthopaedics, Rehabilitation and the Clinical Chemical Laboratory. At present he is Professor in Medical Technology at the University of Rotterdam and in Measurement, Control \& Ergonomics at the University of Delft. 